\title{
Dinâmica de pêndulo físico com ímã na presença de uma bobina
}

\author{
Dynamics of a physical pendulum with a magnet in the presence of a coil \\ Thiago de Cacio Luchese ${ }^{* 1}$, Marcelo Nunes Schneider ${ }^{1}$ \\ ${ }^{1}$ Universidade Federal da Fronteira Sul, Cerro Largo, Rio Grande do Sul, Brasil
}

\begin{abstract}
Recebido em 12 de fevereiro de 2020. Revisado em 23 de março de 2020. Aceito em 24 de março de 2020
Investigamos a dinâmica de um pêndulo físico feito de madeira, suportado por uma estrutura de PVC e tendo um ímã colado em sua extremidade oscilante e cuja oscilação se deu nas proximidades de uma bobina que estava ora em curto, ora aberta. A trajetória pendular, posição em função do tempo, foi levantada com auxílio de análise quadro a quadro de vídeos e foi constatado um maior amortecimento da oscilação quando esta ocorria na presença da bobina em curto. As equações de movimento para o pêndulo foram modeladas e resolvidas, mostrando-se satisfatórias para descrever as dinâmicas observadas e, com isso, utilizadas para fazer uma previsão interessante de interação entre os campos magnéticos permanente e induzido no caso de um sistema análogo ser construído com características construtivas distintas (momento de inércia reduzido e campo de ímã aumentado). Esperamos que o trabalho possa servir de estímulo a investigações quantitativas de sistemas simples e venha a motivar o aprendizado significativo da Mecânica e do Eletromagnetismo.
\end{abstract}

Palavras-chave: Tracker, indução magnética, modelagem teórica.

We investigated the dynamics of a wood physical pendulum, supported by a PVC structure and having a magnet glued to its oscillating end and whose oscillation occurred in the vicinity of a coil that was sometimes in short-circuit, sometimes in open-circuit. The pendulum trajectory, position as a function of time, was drawn with the aid of frame-by-frame video analysis, helping to detect a greater damping of the oscillation in the presence of the coil in short-circuit. The equations of motion for the pendulum were modeled and solved, showing to be satisfactory to describe the observed dynamics and, thus, used to make an interesting prediction of interaction between the permanent and induced magnetic fields in the case of an analogous system to be built with distinctive construction characteristics (reduced moment of inertia and increased magnet field). We hope that the work can serve as a stimulus to quantitative investigations of simple systems and will motivate the significant learning of Mechanics and Electromagnetism.

Keywords: Tracker, magnetic induction, theoretical modeling.

\section{Introdução}

Tornar a Física e sua linguagem quantitativa atraente ao público aprendiz é um grande desafio. Embora a experiência dos professores os leve a um caminho de construção conceitual parcimoniosa, com frequência seccionada em partes para tornar uma primeira abordagem das suas várias subáreas mais suave, constata-se ser muito difícil mostrar a um estudante que essa separação (histórica) de subáreas da Física é uma necessidade, não uma opção. Enquanto ao estudante pode parecer que as informações a ele transmitidas não são conectadas, na realidade a vitória lógica da desconexão idealizada das subáreas clássicas é requisito para a compreensão dos fundamentos da natureza.

Sendo assim, uma das intenções da apresentação desse trabalho é instigar a compreensão em separado de algumas das subáreas estruturantes da Física Clássica, a sa-

*Endereço de correspondência: thiago.luchese@uffs.edu.br ber, a Mecânica e o Eletromagnetismo, para então utilizar essa compreensão na resolução do problema lúdico aqui apresentado. Essa motivação deve nascer naturalmente a partir da atividade desenvolvida e de seus resultados que conectam conceitos, compreensões e intuição advindas dessas duas subáreas.

Outro objetivo do trabalho é, por meio de um experimento simples com uma metodologia objetiva de extração de dados, estimular hábitos como o estabelecimento de reprodutibilidade de uma experiência e seu relato, a construção de um modelo teórico consistente com o corpus investigativo já estabelecido para explicar, a partir deste, os resultados obtidos e, caso haja sucesso no processo de modelagem, o uso do modelo para fazer previsões a respeito de futuras experiências (que virão a servir de teste e melhoramento do modelo).

Na experiência que relatamos, um ímã foi colado na extremidade mais distante do pivô de um pêndulo físico e, em seguida, o pêndulo foi posto a oscilar nas proximidades de uma bobina. A oscilação resultante foi observada com 
a bobina tendo seus terminais distantes um do outro (circuito aberto) e, também, tendo seus terminais unidos (circuito fechado, bobina em curto). Essas duas situações foram filmadas repetidas vezes com um celular e os vídeos resultantes foram analisados com o software livre Tracker 1]. Este, por sua vez, possibilitou o estabelecimento de uma trajetória média (e seu desvio padrão) para cada uma das duas situações distintas investigadas. A existência de interação entre o campo do ímã e o campo da corrente induzida pôde ser observada de modo indireto quando da constatação de um amortecimento mais rápido da oscilação na presença da bobina em curto do que na presença da bobina com terminais livres.

Na seção seguinte (Seção 2 apresentamos o equipamento e alguns detalhes de sua construção e da coleta de dados. Em seguida, na Seção 3, apresentamos o modelo teórico desenvolvido para explicar os dados da experiência bem como a metodologia de ajuste de parâmetros livres adotada para gerar a regressão do modelo aos dados experimentais. Verificada a possibilidade de descrever os dados com o modelo teórico adotado, na Seção 4 fazemos algumas previsões a respeito de dinâmicas interessantes de sistemas análogos a esse e, por fim, na Seção 5 tecemos alguns comentários gerais.

\section{Equipamento e coleta de dados}

Uma haste de madeira de formato paralelepipédico com dimensões aproximadas de (largura $\mathrm{x}$ altura $\mathrm{x}$ comprimento $)=(2 \times 3 \times 15) \mathrm{cm}$ foi lixada com uma microretífica até ficar no formato apresentado na Figura 17 e 1. pectivamente. Em seguida um ímã de neodímio, também paralelepipédico com dimensões (largura x altura $\mathrm{x}$ comprimento $)=(4 \times 10 \times 15) \mathrm{mm}$ foi fixado com cola quente na extremidade achatada da barra tendo sua direção norte-sul magnética coincidente com o comprimento do pêndulo e, também, um furo foi feito na outra extremidade da barra com a finalidade de constituir a posição do pivô de oscilação do que veio a ser o pêndulo físico a ser estudado. Para diminuir o atrito durante a oscilação, inserimos um pequeno rolamento no furo do pivô.

A base do suporte de oscilação foi construída com um corte de madeira paralelepipédico com dimensões $($ largura $\mathrm{x}$ altura $\mathrm{x}$ comprimento $)=(6 \times 1 \times 25) \mathrm{cm}$. Nas laterais dessa madeira um corte circular com serra copo permitiu o encaixe de canos de PVC. Cada um dos canos também foi cortado com serra copo em uma altura tal que permitisse o encaixe de um terceiro pedaço de cano entre eles, este terceiro pedaço vindo a servir de apoio ao pivô do pêndulo físico antes construído. Para facilitar a montagem do sistema, este cano que suportou o pêndulo teve suas laterais retiradas longitudinalmente. Finalmente, a conexão entre pivô e suporte foi sustentada com um parafuso que atravessava o rolamento do pivô do pêndulo físico e tinha suas extremidades inseridas em orifícios previamente efetuados no cano de sustentação

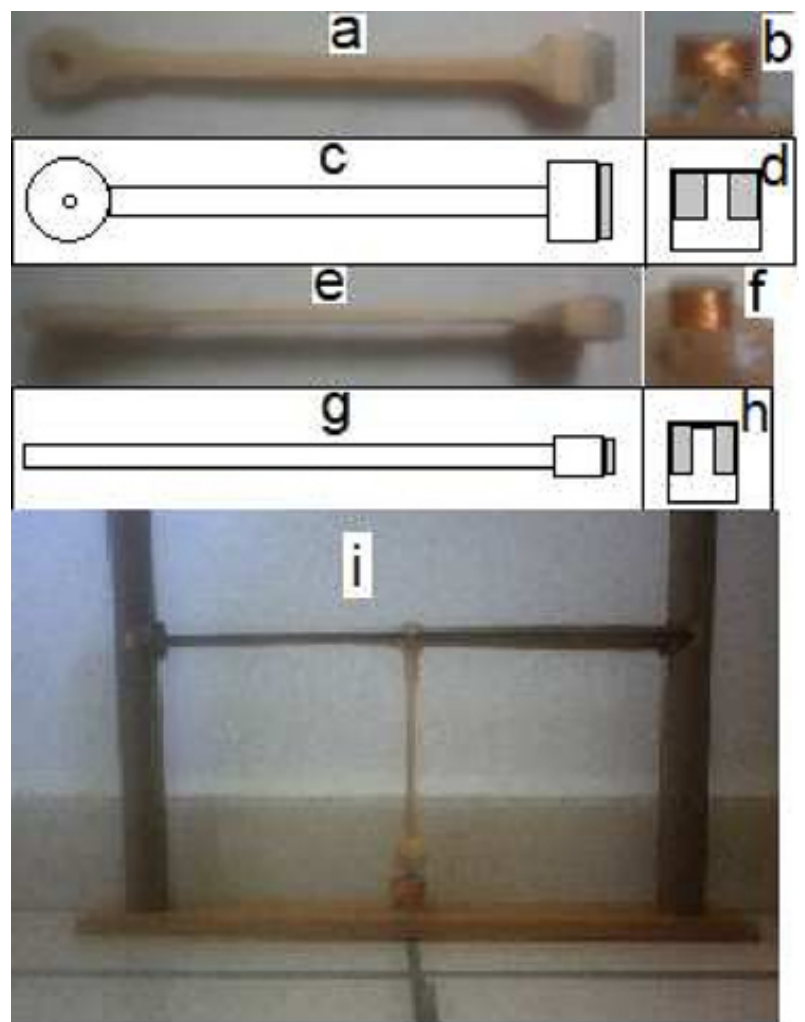

Figura 1: Em a e e pode-se ver o pêndulo construído nos perfis de frente e de lado, antes de ser montado no suporte que permitirá sua oscilação. Também antes de ser acoplada ao suporte, vê-se em $b$ e $f$ a bobina, de frente e de lado, respectivamente. Em $c, d, g$ e h apresenta-se o modelo geométrico adotado na estimativa do momento de inércia do pêndulo. Finalmente, em $i$ o pêndulo montado em seu suporte e já com a bobina logo abaixo da sua posição de equilíbrio estável pode ser visualizado.

do pivô no suporte. O conjunto montado é apresentado na Figura 1.

A bobina do sistema foi construída também tomandose um pequeno pedaço paralelepipédico de madeira com dimensões aproximadas de (comprimento $\mathrm{x}$ largura $\mathrm{x}$ altura $)=(2 \times 2 \times 2) \mathrm{cm}$ e gastando-se com a microretífica até que pudesse acomodar um enrolamento nessa forma assim construída. O resultado final foi um enrolamento, manualmente construído, de aproximadamente 60 espiras dispostas em 6 camadas de 10 espiras cada uma. A bobina pode ser visualizada nas Figuras $1 \mathrm{~b}$ e 1f, de frente e de lado, respectivamente. A bobina foi fixada à base de madeira por meio de duas abraçadeiras de nylon ${ }^{1}$

Posicionado-se um celular a aproximadamente $20 \mathrm{~cm}$ do suporte do pêndulo, tendo o pêndulo no centro da imagem da câmera do celular, foram produzidos 10 vídeos repetidos da oscilação do pêndulo quando a bobina estava com seus terminais separados (circuito aberto) e outros 10 vídeos repetidos da oscilação do pêndulo

${ }^{1}$ Seja notado que não há, em todo o aparato, nenhum material ferromagnético nas proximidades da oscilação, a não ser o rolamento colocado no pivô do pêndulo e o parafuso que o prende ao suporte de cano. Supomos que estes materiais não alteram significativamente a dinâmica do sistema. 
quando a bobina estava com seus terminais unidos (circuito fechado). A oscilação foi iniciada, em cada uma das 20 vezes, deslocando-se o pêndulo da sua posição de equilíbrio com uma régua até que a área do ímã estivesse sem sobreposição com a área das espiras da bobina e, em seguida, soltando-o a partir do repouso. Um ponto azul, bastante visível no pêndulo, foi feito com a finalidade de permitir o traçado automático da trajetória oscilatória com uso do software Tracker, um software livre de análise de vídeos [1] que tem sido utilizado no contexto de práticas de ensino de física 2, 3]. Na Figura 2 apresenta-se um print da tela de trabalho com o software, já com a trajetória automática gerada para um dos vídeos produzidos (visto no canto superior direito da imagem), onde pode-se notar o posicionamento do sistema coordenado com origem coincidente com o pivô de oscilação do pêndulo, eixo $x$ horizontal com sentido positivo para direita e eixo $y$ vertical com sentido positivo para cima (eixos vistos em rosa na Figura 2 .

A Figura 3 apresenta as trajetórias médias obtidas, sendo as barras de erro em cada ponto da trajetória correspondentes ao desvio padrão da média de cada ponto nas trajetórias. Pode-se notar que trata-se de duas dinâmicas efetivamente distintas, dentro da margem de erro experimental acessada pela metodologia, o que evidencia a modificação das forças responsáveis pela dinâmica observada quando da conexão entre os terminais da bobina (fechamento do circuito). O recorte de tempo adotado para análise da dinâmica foi de 10 segundos.

\section{Construindo um modelo}

Assumiremos que a dinâmica pendular com a bobina aberta (terminais separados) - caso 1 - pode ser descrita pela aplicação da Segunda Lei de Newton para rotações de objetos rígidos em torno de eixo fixo (ver [4], capítulos

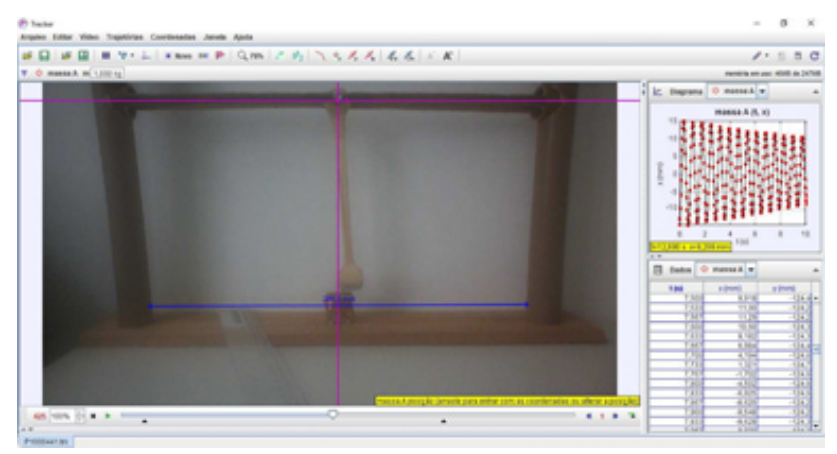

Figura 2: Tela de trabalho do Tracker no ato de levantamento automático das trajetórias de oscilação do pêndulo físico. No canto superior direito pode-se notar o gráfico da coordenada $x$ do ponto de referência (ponto azul previamente feito no pêndulo) como função do tempo enquanto que, no canto inferior direito nota-se os dados numéricos correspondentes ao gráfico dispostos em uma tabela. Esses dados dão origem à trajetória média em cada um dos casos observados e seus respectivos desvios padrão.

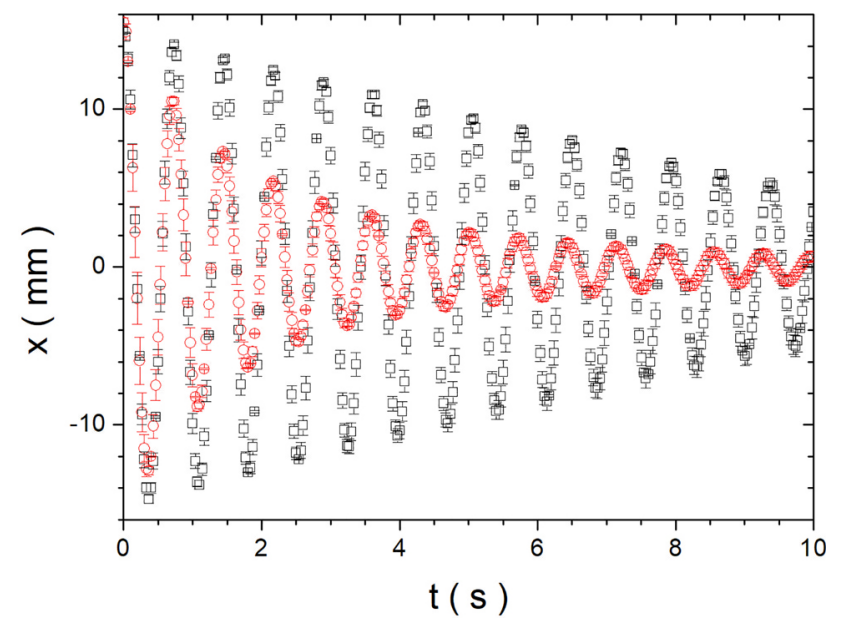

Figura 3: Trajetórias médias de oscilação, conforme dados automáticos gerados pelo Tracker. Os quadrados (pretos) correspondem à oscilação com a bobina tendo terminais separados (circuito aberto) enquanto que os círculos (vermelhos) correspondem à oscilação com a bobina tendo seus terminais unidos (circuito fechado). As barras de erro nas trajetórias correspondem ao desvio padrão da média das dez trajetórias levantadas para cada caso.

10 e 11):

$$
\tau_{g}+\tau_{a}=I \alpha
$$

sendo $\tau_{g}$ o torque gravitacional, $\tau_{a}$ o torque de amortecimento, $I$ e $\alpha$ o momento de inércia e a aceleração angular, respectivamente, do pêndulo físico.

O torque gravitacional, devido ao peso do pêndulo, é dado por

$$
\tau_{g}=-m g l \operatorname{sen} \theta
$$

sendo o sinal negativo o indicativo de se tratar de um torque restaurador, $m$ a massa do pêndulo, $g$ a aceleração gravitacional, $l$ a distância entre o pivô e o centro de massa do pêndulo e $\theta$ o ângulo de deslocamento do pêndulo desde a sua posição de equilíbrio, sendo positivo no sentido anti-horário de rotação.

O torque de amortecimento será admitido como diretamente proporcional à velocidade angular, sendo modelado como

$$
\tau_{a}=-\beta \frac{d \theta}{d t}
$$

$\operatorname{com} \beta$ a constante de proporcionalidade entre a velocidade angular e o torque total de amortecimento.

Com as expressões (2) e (3) substituídas na equação de movimento, equação (1), após rearranjo de termos, obtém-se

$$
\frac{d^{2} \theta}{d t^{2}}+\frac{\beta}{I} \frac{d \theta}{d t}+\frac{m g l}{I} \operatorname{sen} \theta=0
$$

Considerando que a largura da bobina e do ímã são, respectivamente, $1,6 \mathrm{~cm}$ e $1,5 \mathrm{~cm}$, que a distância entre o pivô e o ímã (o comprimento total do pêndulo $L$ ) é de $15 \mathrm{~cm}$ e que as dinâmicas eram iniciadas com o ímã totalmente fora de sobreposição com a bobina, sendo que sua posição de equilíbrio é totalmente sobreposto a 
esta, é possível afirmar que os ângulos de oscilação se mantiveram inferiores a

$$
\operatorname{sen} \theta<\frac{1,6 / 2+1,5 / 2}{15}=0,10 \Rightarrow \theta \leq 6^{\circ}
$$

permitindo o uso da aproximação

$$
\operatorname{sen} \theta=\frac{x}{l+d} \simeq \theta
$$

com $x$ a posição do ponto azul do pêndulo seguida pelo Tracker durante a dinâmica e $d$ a distância entre o centro de massa e o ponto azul do pêndulo. Levando essa aproximação na equação de movimento (4), substituindo a variável dinâmica $\theta$ por $x$, é possível escrever

$$
\frac{d^{2} x}{d t^{2}}+\frac{\beta}{I} \frac{d x}{d t}+\frac{m g l}{I} x=0
$$

cuja solução é dada por (ver [4], capítulo 15)

$$
x(t)=x_{0} e^{-\frac{\beta t}{2 I}} \cos \omega t
$$

com

$$
\omega=\sqrt{\frac{m g l}{I}-\frac{\beta^{2}}{4 I^{2}}}
$$

e $x_{0}$ a posição inicial do pêndulo.

A equação (6), a depender da combinação de valores de seus parâmetros, apresenta três regimes dinâmicos bastante característicos, a saber: (i) dinâmica subamortecida, que acontece quando $\frac{\beta^{2}}{4 I^{2}}<\frac{m g l}{I}$, sendo caracterizada pela existência de oscilações cuja amplitude decresce exponencialmente à medida que o tempo passa; (ii) dinâmica de amortecimento crítico, quando $\frac{\beta^{2}}{4 I^{2}}=\frac{m g l}{I}$, resultando em um movimento ausente de oscilações e com tempo de aproximação ao equilíbrio caracterizado apenas pelo parâmetro de amortecimento $\frac{\beta}{2 I}$; (iii) dinâmica superamortecida, que acontece quando $\frac{\beta^{2}}{4 I^{2}}>\frac{m g l}{I}$, também apresentando ausência de oscilações, porém levando um tempo mais longo que na dinâmica (ii) para atingir o equilíbrio. Desse modo, a dinâmica que estamos investigando nesse trabalho é a de subamortecimento.

Embora fosse conhecida a solução analítica da equação (6), com a finalidade de termos um programa prévio para o próximo passo de modelagem do sistema, ela foi numericamente resolvida. Um programa com o método numérico Runge-Kutta de quarta ordem foi construído para resolvê-la [5], dando-se as condições iniciais de posição $x_{0}=15 \mathrm{~mm}$ e velocidade $\left.\frac{d x}{d t}\right|_{0}=0$ e assumindo-se como parâmetros livres para ajuste as constantes multiplicativas nos dois últimos termos da equação. O método de escolha dos parâmetros livres foi o dos mínimos quadrados, fazendo-se uma varredura no espaço (bidimensional) de parâmetros: para cada par de parâmetros a equação de movimento (6) era resolvida, fornecendo uma trajetória correspondente ao modelo adotado, e, em seguida, a soma dos quadrados das diferenças entre as coordenadas experimentais e teóricas era efetuada; o par de parâmetros que fornecesse a menor soma quadrática era assumido como aquele que melhor ajustava a curva resultante do modelo com os dados experimentais.

Para os dados apresentados na Figura 3 , quadrados pretos, foi obtido

$$
\frac{\beta}{I}=4,10 \mathrm{~s}^{-1} \quad \text { e } \quad \frac{m g l}{I}=83,0 \mathrm{~s}^{-2} .
$$

Considerando que $m=10,00 \mathrm{~g}, g=979 \mathrm{~cm} / \mathrm{s}^{2}$ e $l=10$ $\mathrm{cm}$, tratam-se de duas equações e duas incógnitas, a saber, $\beta$ e $I$. Resolvendo, encontramos o momento de inércia $I$ e a constante de amortecimento $\beta$

$$
I=11,6 \times 10^{4} \mathrm{~g} \mathrm{~mm}^{2} \quad \beta=47,4 \times 10^{4} \mathrm{~g} \mathrm{~mm}^{2} / \mathrm{s} .
$$

Apenas a critério de comparação, modelando o pêndulo físico como sendo constituído de um disco e três paralelepípedos, conforme geometria indicada na Figura 1. e 1. (disco com diâmetro de $19 \mathrm{~mm}$, espessura de 4 $\mathrm{mm}$, diâmetro do furo em seu centro de $7 \mathrm{~mm}$; paralelepípedos com dimensões (comprimento x largura x altura), da esquerda para a direita e em milímetros, de (115x7x4), $(11 \times 19 \times 10)$ e $(4 \times 16 \times 10)$, lembrando que o comprimento é a dimensão que se dá ao longo da distância até o pivô), e considerando a densidade da madeira e do ímã dadas por $\rho_{m}=1,05 \mathrm{~g} / \mathrm{cm}^{3}$ e $\rho_{i}=5,0 \mathrm{~g} / \mathrm{cm}^{3}$, respectivamente, pode-se calcular o momento de inércia em relação ao pivô como tendo o valor $I=12,1 \times 10^{4} \mathrm{~g} \mathrm{~mm}^{2}$, em boa concordância com o valor de ajuste obtido via mínimos quadrados.

As curvas resultantes da média de vídeos para o caso de bobina aberta e sua correspondente oriunda do ajuste de mínimos quadrados são apresentadas na Figura 4 É possível notar que existiram coeficientes capazes de tornar a curva oriunda do modelo teórico compatível (quase sobreposta) aos pontos experimentais, de modo que temos, assim, o indicativo de que o modelo teórico adotado é suficiente para descrever o fenômeno observado.

Para o caso da bobina com terminais conectados (circuito fechado) assumimos que a dinâmica observada seria resultante, também, da aplicação da Segunda Lei de Newton para rotações

$$
\tau_{g}+\tau_{a}+\tau_{b}=I \alpha
$$

onde foi acrescido o torque $\tau_{b}$ na equação (1), devido à presença da bobina em curto e, ainda, têm-se os torques $\tau_{g}$ e $\tau_{a}$ obtidos das expressões 2 e (3), respectivamente.

Para simplificar (muito) o efeito da interação da bobina com o ímã oscilante consideramos o torque $\tau_{b}$ sendo resultante da aplicação de uma força de intensidade $F$ (a ser modelada logo em seguida) diretamente sobre o centro de massa do ímã, tendo direção vertical e sentido dependente da posição e da velocidade do ímã, conforme considerações subsequentes irão indicar. Com isso pode-se escrever

$$
\tau_{b}=F L \operatorname{sen} \theta
$$

e a equação de movimento nesse caso, após alguns rearranjos, pode ser escrita como

$$
\frac{d^{2} x}{d t^{2}}+\frac{\beta}{I} \frac{d x}{d t}+\left(\frac{m g l}{I}-\frac{F L}{I}\right) x=0
$$




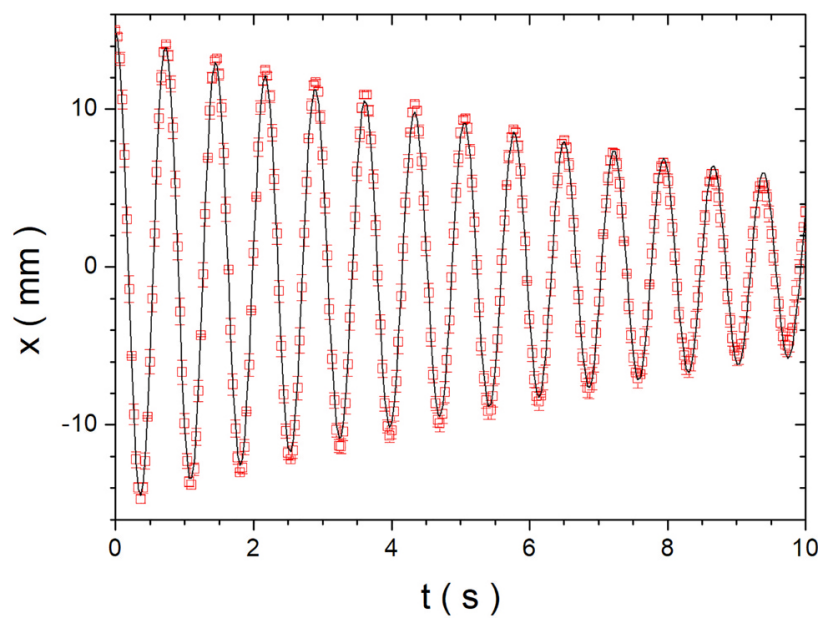

Figura 4: Os quadrados vermelhos abertos correspondem à trajetória média obtida dos vídeos de oscilação do pêndulo quando os terminais da bobina estão separados um do outro (circuito aberto), as barras de erro correspondendo aos respectivos desvios padrão de cada valor médio de posição para aquele tempo de dinâmica. A linha sólida preta resulta da resolução numérica da equação (6) com os seguintes valores para as constantes presentes na equação: $\frac{\beta}{I}=4,10 \mathrm{~s}^{-1}$ e $\frac{m g l}{I}=83,0 \mathrm{~s}^{-2}$.

onde foi considerada válida a aproximação dada pela equação (5).

Considerando que a força $F$ deverá ser (ver [4], capítulos 28 a 30): (i) proporcional à corrente $i$ induzida na bobina; (ii) proporcional ao campo $B$ do ímã; e (iii) dependente da posição do ímã em relação à bobina, posição essa associada à posição $x$ seguida pelo Tracker devido à rigidez do pêndulo, sendo máxima diretamente acima da bobina $(x=0)$ e caindo a zero muito rapidamente quando o ímã deixa de estar sobreposto à essa; propõe-se o seguinte modelo (a dois parâmetros) para o cálculo da intensidade da força $F$ :

$$
F=-K i B e^{-2\left(\frac{x}{b}\right)^{2}}
$$

com $K$ e $b$ sendo constantes a serem determinadas por ajustes de mínimos quadrados, à maneira do efetuado no caso em que determinamos o momento de inércia e a constante de amortecimento anteriormente.

Deve-se notar que, com o polo norte do ímã apontando para o pivô do pêndulo, a convenção de sinais adotada para a corrente foi a seguinte: positiva no sentido antihorário quando a bobina é vista de cima.

Aparece aqui a necessária associação entre os conceitos e compreensões de subáreas distintas da Física, conforme mencionadas na introdução: os conceitos e compreensões apenas de Mecânica não são suficiente para descrever a dinâmica pendular, uma vez que a determinação da força resultante sobre o pêndulo invocará a determinação da corrente (induzida) na bobina, ou seja, conceitos e compreensões do Eletromagnetismo; por sua vez, conforme será visto logo adiante, a determinação da corrente na bobina invocará informações a respeito da dinâmica do pêndulo (posição e velocidade), dinâmica esta de- terminada pelos conceitos e compreensões da Mecânica. Ou seja: ambas as subáreas da Física, com seus conceitos e compreensões, são necessárias para modelar essa dinâmica.

A corrente será determinada pela identificação e quantificação de forças eletro-motrizes e resistências no circuito formado unicamente pela bobina. Chamando de $\epsilon$ a força eletromotriz induzida pelo movimento do pêndulo, $R$ a resistência do fio que compõe o enrolamento da bobina, e $\Lambda$ a indutância da bobina, a equação que relaciona essas grandezas na determinação da dependência da corrente $i$ com o tempo é escrita como

$$
\epsilon-R i-\Lambda \frac{d i}{d t}=0
$$

Um instantâneo de áreas sobrepostas do ímã e da bobina é mostrado na Figura 5 para facilitar a estimativa da força eletromotriz induzida devido à movimentação do ímã logo acima da bobina. A área indicada com pontos finos, mais à esquerda, corresponde a área do ímã oscilante logo acima da bobina. A área com fundo liso, cujo centro coincide com a origem do eixo $x$ indicado, corresponde à área da bobina, fixa nesta posição. Considerando $x$ a coordenada do centro da área do ímã oscilante sobre a bobina (no instantâneo da foto $x<0$ ), pode-se notar que a área sobreposta $\left(A^{\prime}\right.$, indicada por um fundo preenchido com pontos espessos) é dada por

$$
A^{\prime}= \begin{cases}(\bar{b}-x) c & \text { se } x \geq 0 \\ (\bar{b}+x) c & \text { se } x<0\end{cases}
$$

em que $c$ e $\bar{b}$ correspondem à largura e ao comprimento da bobina e do ímã, considerados iguais.

Devido à proximidade entre o ímã e a bobina durante a oscilação, pode-se considerar que a intensidade do campo magnético do ímã é constante e igual a $B$ em toda a sua travessia por sobre a bobina e, com isso, pode-se estimar a força eletromotriz induzida devido à variação de fluxo por meio da seguinte expressão

$$
\epsilon=B c \operatorname{sgn}(x) \frac{d x}{d t}
$$

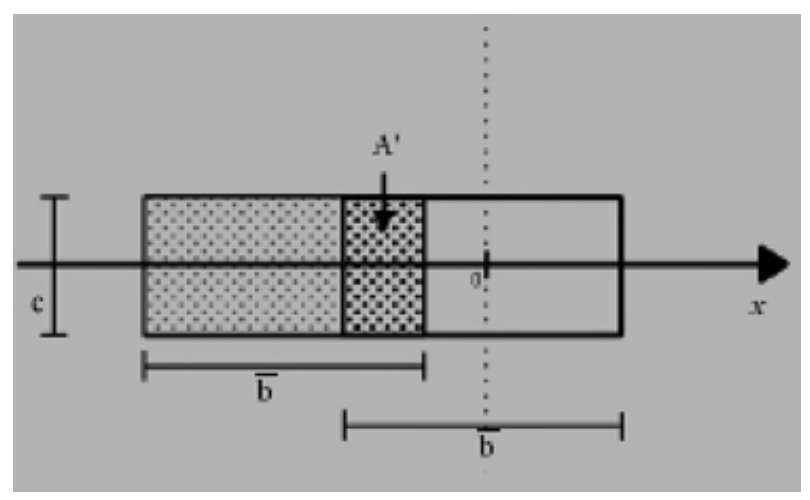

Figura 5: Esquema de sobreposição de áreas (fundo com pontos espessos) da bobina (fundo liso) e do ímã (fundo com pontos finos) em um instante do movimento do ímã logo acima da bobina. 
$\operatorname{com} \operatorname{sgn}(x)$ uma função que extrai apenas o sinal de $x$

$$
\operatorname{sgn}(x)= \begin{cases}+1 & \text { se } x>0 \\ 0 & \text { se } x=0 \\ -1 & \text { se } x<0\end{cases}
$$

Substituindo a força eletromotriz 16 na equação 15 , após rearranjo, escreve-se

$$
\frac{d i}{d t}+\frac{R}{\Lambda} i-\frac{B c}{\Lambda} \operatorname{sgn}(x) \frac{d x}{d t}=0
$$

onde é possível notar, explicitamente, que a determinação da corrente dependerá da determinação da posição e da velocidade do pêndulo.

Desse modo, o modelo adotado para descrição do movimento pendular na presença da bobina em curto resulta no seguinte sistema de duas equações diferenciais acopladas

$$
\begin{aligned}
\frac{d^{2} x}{d t^{2}}+\frac{\beta}{I} \frac{d x}{d t}+\left(\frac{m g l}{I}+\frac{K B L}{I} i e^{-2\left(\frac{x}{b}\right)^{2}}\right) x & =0 \\
\frac{d i}{d t}+\frac{R}{\Lambda} i-\frac{B c}{\Lambda} \operatorname{sgn}(x) \frac{d x}{d t} & =0
\end{aligned}
$$

em que a primeira das equações resultou da substituição do modelo adotado para quantificar a intensidade da força entre bobina e ímã, equação (14), na equação (13) e a segunda é a equação (17).

Assumindo que cada combinação de constantes nas equações obtidas fornece um parâmetro livre para ajuste na resolução dessas duas equações acopladas e considerando os valores já obtidos de $\frac{\beta}{I}$ e $\frac{m g l}{I}$, obtemos um sistema com quatro parâmetros a serem ajustados, a saber, $\frac{K B L}{I}, b, \frac{R}{\Lambda}$ e $\frac{B c}{\Lambda}$.

Procedendo de forma análoga ao caso em que a bobina estava aberta (circuito aberto), fixam-se 3 dos 4 parâmetros, sucessivamente, variando-se um dos parâmetros e permitindo que ele fique fixo naquele valor que minimiza o quadrado da soma das diferenças entre os valores obtidos da solução numérica e aqueles experimentais, recursivamente, até que nenhum deles tenha valor alterado (autoconsistência). Desse modo, foram obtidos os seguintes valores para os parâmetros das equações diferenciais:

$$
\begin{aligned}
\frac{K B L}{I} & =4,19 \times 10^{3} \mu \mathrm{A}^{-1} \mathrm{~s}^{-2} \\
b & =11,5 \mathrm{~mm} \\
\frac{R}{\Lambda} & =49,4 \mathrm{~s}^{-1} \\
\frac{B c}{\Lambda} & =4,52 \mu \mathrm{Amm} \mathrm{mm}^{-1}
\end{aligned}
$$

Estes, por sua vez, considerando o conhecimento das grandezas $L=140 \mathrm{~mm}, I=11,6 \times 10^{4} \mathrm{~g} \mathrm{~mm}^{2}, R=1,1$ $\Omega$ (determinado diretamente com um multímetro) e $c=$
10,0 mm , podem ser resolvidos no campo do ímã $B$, na indutância da bobina $\Lambda$ e nos parâmetros que modelam a força da bobina sobre o ímã ( $K$ e $b$, equação (14)), para fornecer

$$
\begin{aligned}
B & =10,1 \mathrm{mT} \\
K & =3,42 \times 10^{11} \mathrm{~mm} \\
\Lambda & =22,3 \mathrm{mH} \\
b & =11,5 \mathrm{~mm} .
\end{aligned}
$$

A Figura 6 apresenta os dados experimentais (quadrados vazios vermelhos) juntamente com a curva oriunda da resolução numérica das equações acopladas (18) e (19) (curva sólida preta). A concordância entre as dinâmicas é, mais uma vez, indicativo de que o modelo é suficiente para descrever a dinâmica observada.

Com a verificação de que o modelo é capaz de fornecer uma descrição quantitativa da dinâmica observada apresenta-se, a seguir, algumas previsões interessantes do modelo.

\section{Previsões do modelo}

Embora a corrente não tenha sido determinada diretamente, os resultados da resolução numérica das equações acopladas fornecem seu comportamento como função do tempo. Apontamos esse comportamento da corrente, juntamente com o comportamento da posição, em função do tempo para dois casos particulares.

O primeiro dos casos, Figura 7 a, trata da situação correspondente à Figura 6] resolvida na seção anterior.

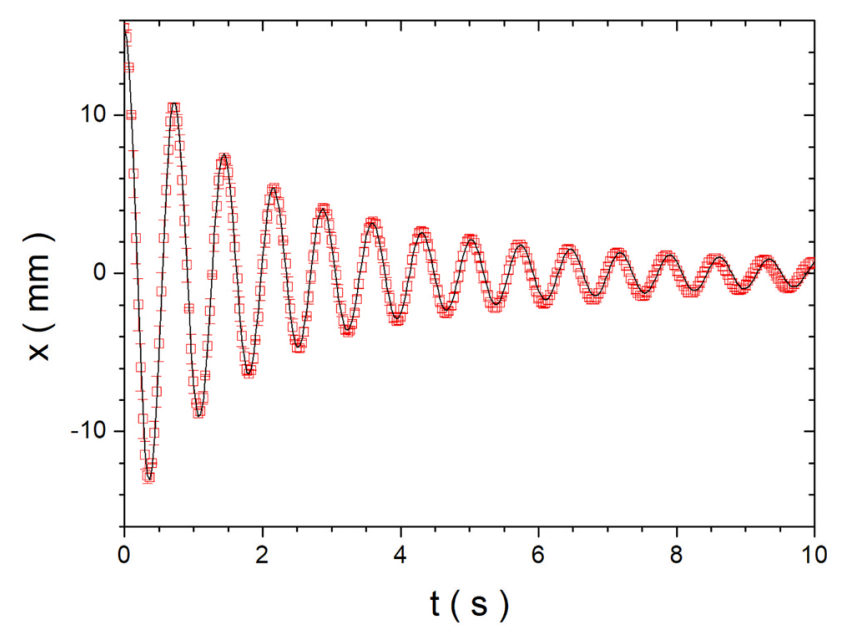

Figura 6: Os quadrados vermelhos abertos correspondem à trajetória média obtida dos vídeos de oscilação do pêndulo quando os terminais da bobina estão unidos um ao outro (circuito fechado), as barras de erro correspondendo aos respectivos desvios padrão de cada valor médio de posição para aquele tempo de dinâmica. A linha sólida preta resulta da resolução numérica das equações (18) e (19) com os seguintes valores para as constantes presentes nessas equações: $\frac{\beta}{I}=4,10 \mathrm{~s}^{-1}, \frac{m g l}{I}=83,0 \mathrm{~s}^{-2}$, $\frac{K B L}{I}=4,19 \times 10^{3} \mu \mathrm{A}^{-1} \mathrm{~s}^{-2}, b=11,5 \mathrm{~mm}, \frac{R}{\Lambda}=49,4 \mathrm{~s}^{-1} \mathrm{e}^{\prime}$ $\frac{B c}{\Lambda}=4,52 \mu \mathrm{A} \mathrm{mm}{ }^{-1}$. 
O destaque a ser dado para esse caso é que o intervalo de tempo necessário para haver inversão do sentido de corrente, quando o ímã passa de posições $x$ positivas para negativas, é muito menor que o período de oscilação do pêndulo e pode, nesse caso, ser considerada uma inversão instantânea em um possível modelo de solução analítica ao problema. Deve-se notar que esse tempo de inversão depende dos parâmetros mecânicos e eletromagnéticos do sistema, em especial do momento de inércia e da indutância da bobina.

O segundo dos casos, Figura $7 \mathrm{p}$, apresenta uma possível "colisão" do campo do ímã com o campo que ele mesmo induziu. Embora tal colisão esteja sempre acontecendo, para qualquer conjunto de parâmetros, nesse caso específico há uma reversão da velocidade do ímã antes mesmo de ele atingir a posição de equilíbrio! Ou seja, o ímã bateu no campo induzido e retrocedeu, assim como uma bola batendo na parede só que levando um tempo maior para reverter a velocidade (colisão macia, por assim dizer). Tal dinâmica foi construída com os seguintes parâmetros nas equações de movimento 18 e 19 : $\frac{\beta}{I}=4,10 \mathrm{~s}^{-1}$, $\frac{m g l}{I}=83,0 \mathrm{~s}^{-2}, \frac{K B L}{I}=20 \times 10^{3} \mu \mathrm{A}^{-1} \mathrm{~s}^{-2}, b=11,5 \mathrm{~mm}$, $\frac{R}{\Lambda}=10 \mathrm{~s}^{-1}$ e $\frac{B c}{\Lambda}=4,52 \mu \mathrm{A} \mathrm{mm} \mathrm{m}^{-1}$. Notar que, efetivamente, esses parâmetros correspondem à escolha de um ímã cinco vezes mais intenso que aquele utilizado aqui e uma bobina com indutância cinco vezes maior que aquela que construímos. A possibilidade de exploração lúdica desse sistema para falar de Mecânica, Eletromagnetismo e Termodinâmica (a energia dissipada se torna calor!) visualizando a fenomenologia que acopla as subáreas da Física Clássica é muito rica!

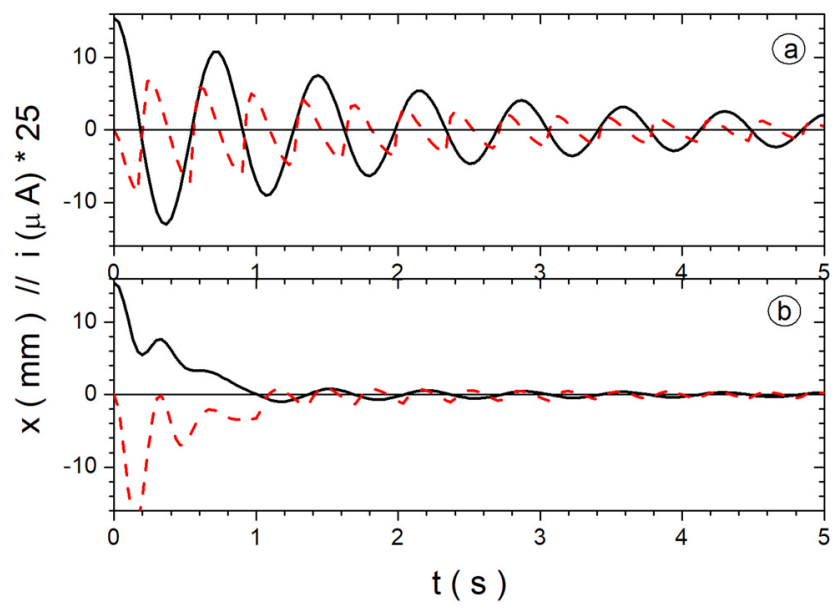

Figura 7: Corrente (linha vermelha tracejada) e posição (linha preta sólida) em função do tempo para: (a) conjunto de parâmetros que ajustaram a trajetória experimental da Figura 6. (b) conjunto de parâmetros que possibilita a visualização de uma "colisão" de ímãs: o permanente e o induzido. Esse segundo exemplo explicita que um controle de parâmetros pode ser utilizado de maneira lúdica para abordar que uma "colisão" não necessariamente implica contato físico, é possível haver uma "colisão" de campos.

\section{Considerações finais}

Exploramos um sistema de relativa facilidade de montagem que permite exploração qualitativa e quantitativa de conexões entre a Mecânica, o Eletromagnetismo e, caso seja um pouco mais explorado, a Termodinâmica. Essa conexão lúdica entre as áreas pode servir de ponte para discussões acerca da metodologia investigativa adotada nas ciências factuais bem como de justificativa e motivação para o estudo detalhado em separado de cada uma das subáreas da Física Clássica.

Embora saibamos que o cálculo da variação de fluxo magnético responsável pela interação entre o ímã e a bobina, necessário para estimar a força entre esses elementos do sistema analisado, possa ser efetuado [6], as complicações inerentes a esse processo de quantificação motivou-nos a modelar a força resultante entre ímã e bobina por meio de uma expressão mais simples mas que contivesse os ingredientes qualitativos do sistema. A possibilidade de ajustar o resultado experimental a partir do modelo simplificado pode, também, motivar estudantes de todos os níveis a não recuarem diante de uma possível dificuldade de exploração de dados levantados em uma "brincadeira" e, sim, explorar os dados com as ferramentas que possui.

Mesmo com o modelo simplificado obtido, o acoplamento entre as equações dinâmicas obtidas pelo modelo leva à utilização de resolução numérica dessas equações, ferramenta também atualmente necessária a uma boa formação inicial em cursos de Física.

O ponto de partida de toda a quantificação de dados foi o sistema mecânico. No entanto, após a modelagem da força resultante entre ímã e bobina e a consequente resolução numérica das equações advindas do modelo, há necessidade de identificar as unidades associadas às grandezas que saem naturalmente do processo de resolução: quais são as unidades apropriadas de campo magnético, corrente e resitência elétrica, indutância, constante de acoplamento $K$, entre outras? A resposta a essa pergunta conduz, naturalmente, a discussões a respeito da importância do domínio de análise dimensional e sistemas de unidades em Física.

É importante destacar que este trabalho complementa outros presentes na literatura, o que evidencia a riqueza da fenomenologia presente nesse aparato simples. Para citar alguns, a referência [7] apresenta uma explanação a respeito das correntes de Foucault e como essas atuam no amortecimento de um pêndulo físico com extremidade metálica posto a oscilar na presença de um campo magnético. Já a referência [8], além de conter uma amostra de referências interessantes que tratam de pêndulos que oscilam na presença de campos magnéticos estáticos e variáveis, apresenta uma análise detalhada, numérica e experimental, de um pêndulo duplo com forçamento magnético. Por fim, vale chamar a atenção ao sistema de pêndulo com ímã na presença de bobina alimentada por corrente alternada tendo linhas de campo paralelas, pêndulo esse que apresenta um número discreto de amplitudes de oscilação, número este dependente da frequência 
da corrente que passa pela bobina. Esse sistema é chamado de pêndulo de Doubochinski [9].

Esperamos que esse trabalho contribua com o ensino de Física, seja qualitativa-conceitual ou quantitativametodológica, em todos os níveis de ensino e que, também, devido à sua riqueza de possibilidades exploratórias, motive o domínio de técnicas de exploração sistemática de eventos interessantes do dia-a-dia.

\section{Agradecimentos}

Os autores são gratos aos avaliadores por terem chamado a atenção ao fato de a bibliografia a respeito do tema ser abundante.

\section{Referências}

[1] Tracker, disponível em <https://physlets.org/tracker/>, acessado em 11/07/2019.

[2] W. Bonventi Jr. e N. Aranha, Revista Brasileira de Ensino de Física 37, 2504 (2015).

[3] A.G. Bezerra Jr., L.P. Oliveira, J.A. Lenz e N. Saavedra, Caderno Brasileiro de Ensino de Física 29, 469 (2012).

[4] D. Halliday, R. Resnick e J. Walker, Fundamentos de Física (LTC Editora Ltda, Rio de Janeiro 2016).

[5] P.L. DeVries, A first course in Computational Physics (John Wiley and Sons, New York, 1994).

[6] J.D. Jackson, Classical Electrodynamics (John Wiley and Sons, New York, 1998).3ed.

[7] P.V.S. Souza, C.J.V. Silva and W.F. Balthazar, Revista Brasileira de Ensino de Física 41, e20180257 (2019).

[8] M. Wojna, G. Wasilewski, J. Awrejcewicz e A. Wijata, em: Mechatronics 2017 - Ideas for Industrial Applications editado por J. Świder, S. Kciuk e M. Trojnacki (Springer, Cham, 2017), p. 455.

[9] Doubochinski's pendulum, disponível em <https://en.wikipedia.org/wiki/Doubochinski \%27s_pendulum>, acessado em 23/03/2020. 